\title{
Reflexive blood pressure and heart rate responses of spontaneous hypertensive rats to electric shock
}

\author{
DANIEL C. HATTON, THOMAS MAHALIK, and ROBERT D. FITZGERALD \\ Department of Medical Psychology, Oregon Health Sciences University, Portland, Oregon 97201
}

\begin{abstract}
An examination was made of the blood pressure (BP) and heart rate (HR) responses of two groups of Okamoto-Aoki spontaneously hypertensive (SHR) rats and two groups of Wistar/ Kyoto (WKY) genetic control rats to unsignaled 1.5- or 5.0-sec 1.3-mA ac electric shocks applied through chest electrodes. Shock elicited brief pressor BP responses in both strains, followed by a depressor reaction in the WKY groups and a less precipitous fall in pressure in the SHR groups. Heart rate was generally accelerative in both strains. The results suggested that the SHR groups may have been less able to reflexively inhibit sympathetic output than were the WKY groups.
\end{abstract}

In a previous study of pharmacologic blockade of autonomic nervous system functioning during aversive classical conditioning (Hatton, Buchholz, \& Fitzgerald, 1981), it was found that spontaneously hypertensive rats (SHR) demonstrated larger pressor blood pressure (BP) CRs and URs than did genetic control Wistar/Kyoto (WKY) rats. It was also noted that the WKY rats showed substantial depressor activity as part of the CRs and URs, whereas the SHRs did not. Only when alpha-adrenergic activity was blocked by phentolomine did the SHR strain exhibit clear vasodilatory depressor BP changes, and those occurred in response to the shock US. The pattern of the outcomes was in keeping with other findings suggesting that SHRs may have higher than normal sympathetic reactivity (Hallback \& Weiss, 1977) and impaired vasodilation (Yamaguchi \& Kopin, 1980).

In evaluating the absence of SHR depressor responses to shock in the previous study (Hatton et al., 1981), it should be noted that BP was averaged over relatively long 2 -sec intervals beginning approximately $1 \mathrm{sec}$ after termination of the .5 -sec shock. It is conceivable that an assessment of BP in shorter measurement intervals, including the shock period itself, would have revealed the presence of depressor activity in the SHR strain. The current experiment provides information on the BP and heart rate (HR) responses of SHR and WKY rats to unsignaled shocks in successive .5-sec intervals beginning with the onset of shock and for several seconds following shock. Shock duration was varied in separate groups of rats to determine whether the dynamics of the BP and HR

This research was supported by Training Grant 5 T32 HL07332 from the National Heart, Lung, and Blood Institute. Address reprint requests to Robert D. Fitzgerald, Department of Medical Psychology, University of Oregon Health Sciences Center, Portland, Oregon 97201. changes to shock onset would be influenced by the continued presence of shock.

\section{METHOD}

\section{Subjects}

Fifteen naive, male, Okamoto-Aoki spontaneously hypertensive and 16 naive, male, Wistar/Kyoto genetic control rats were used. Eight of the SHRs and all of the WKYs were obtained from Taconic Farms, Germantown, New York; the remaining seven SHRs were obtained from Charles River Laboratories (Wilmington, Mass.). The rats ranged in age from 12-16 weeks and were individually caged under conditions of a 12-h light/dark cycle, with food and water available ad lib. The rats were tested during the light portion of the cycle.

\section{Apparatus}

Restraint was provided by an inverted U-shaped plastic holder with adjustable inserts at both ends that were positioned to hold the rats securely in place. The restrainer was located in an Industrial Acoustics Corporation sound-isolation chamber equipped with an exhaust fan and a 40-W houselight that provided continuous illumination. Additional masking of unwanted sounds was provided by white noise measuring $75 \mathrm{~dB}$ SPL on the $C$ scale of an Ivie Electronics, Inc., audio spectrum analyzer and presented through a $12.5-\mathrm{cm}$ speaker attached to the ceiling of the chamber.

Arterial BP was recorded from the abdominal aorta on a Grass Model-5 polygraph using a Statham P23-Db pressure transducer and a Grass-5Pl preamplifier. Mean systemic measures of BP were provided by an on-line system that continuously converted BP levels to a proportional frequency. The system operated by feeding the output of the Grass 5Pl preamplifier to a Tektronix AM 502 differential amplifier, which in turn was connected to a Tektronix DC 501 function generator that served as an analog BP to digital converter. A continuous train of pulses from the function generator was increased linearly by the positive voltages (above zero, BP increases) and decreased linearly by the negative voltages (below zero, BP decreases) coming from the differential amplifier. The pulses were detected by a Schmidt trigger and totaled electronically, and the numbers were punched on paper tape.

The ECG was recorded on a second channel of the Grass polygraph. Individual heartbeats were sensed from the analog BP signal using a Massey-Dickinson resistive shift trigger feeding an electronic counting circuit. Heartbeat totals were punched, along with BP, on paper tape. 
The US was a $1.3-\mathrm{mA} 60-\mathrm{Hz}$ ac electric shock provided by a Grason-Stadler 6070B shock generator and delivered through 20-ga hypodermic needles inserted under the skin on either side of the rat's thoracic cavity. Shock intensity was determined by measuring the voltage drop across a fixed resistor in series with the rat. Trials were initiated automatically by a film-tape programmer, with stimulus events occurring within a trial controlled by solidstate electronics.

\section{Procedure}

Surgery was performed under general anesthesia provided by an ip injection of $40 \mathrm{mg} / \mathrm{kg}$ of body weight of sodium pentobarbital. A $3.0-\mathrm{cm}$ midline incision was made in the abdominal wall, exposing the abdominal cavity. Facae were separated to the depth of the aorta at the branching of the left renal vein. A catheter, consisting of a section of PE-90 tubing joined to a section of PE-10 tubing, was passed subcutaneously down the back of the neck and through the left body wall and was anchored to a major back muscle approximately $3 \mathrm{~mm}$ lateral to the aorta. The catheter was filled with heparinized saline, and approximately $7 \mathrm{~mm}$ of the PE-10 section was inserted into the aorta in a downstream position such that the tip was below the renal arteries. No ties were taken around the catheter, permitting free circulation of blood to the extremities. The abdominal wound was closed, and the free end of the catheter was anchored to the base of the neck with sutures. The animals were given a minimum of 3 days (most animals received 4 days) to recover from the surgery before they were used in the study. On each day, the catheter was flushed with a heparin solution.

The design of the study was a $2 \times 2$ factorial; one of the factors was rat strain and the other shock duration $(1.5 \mathrm{vs} .5 .0 \mathrm{sec})$. The eight SHRs obtained from Taconic Farms received the 1.5-sec shock, while the seven SHRs purchased at a later time from Charles River received the 5-sec shock. The WKYs were distributed equally between the two duration groups. All animals were given a 15-min adaptation period in the restrainer followed by 12 unsignaled shock-alone trials. The time between trials varied semirandomly among 120-, 150-, and 180-sec intervals (mean $=150 \mathrm{sec}$ ).

Blood pressure and HR were recorded in 19 consecutive intervals within each trial. The first interval was $6 \mathrm{sec}$ long and was located immediately prior to the onset of shock. The rest of the intervals were $.5 \mathrm{sec}$ long and occurred in sequence during shock and following shock termination. Blood pressure in $\mathrm{mm} / \mathrm{Hg}$ and $\mathrm{HR}$ in beats per minute (bpm) during the 6-sec preshock interval were subtracted from the BP and HR levels during each .5-sec interval to form difference scores.

\section{RESULTS}

\section{Blood Pressure}

Although not plotted, trial blocks (four blocks of three trials each) were included as a factor in all analyses of variance.

The BP responses of the four groups, averaged over the 12 shock-alone trials, are shown in Figure 1. Both SHR groups showed an initial pressor change to shock, whereas the two WKY groups showed an initial pressor change followed by a more sustained depressor reaction. In the SHR 5-sec group, BP reached a peak during the early part of shock and then returned toward baseline even though shock was still being applied. The pressor change was more sustained in the 5.0-sec group than in the 1.5-sec group. Following the offset of shock, both SHR groups displayed a second pressor reaction that was nearly as large as the first pressor peak, especially in the case

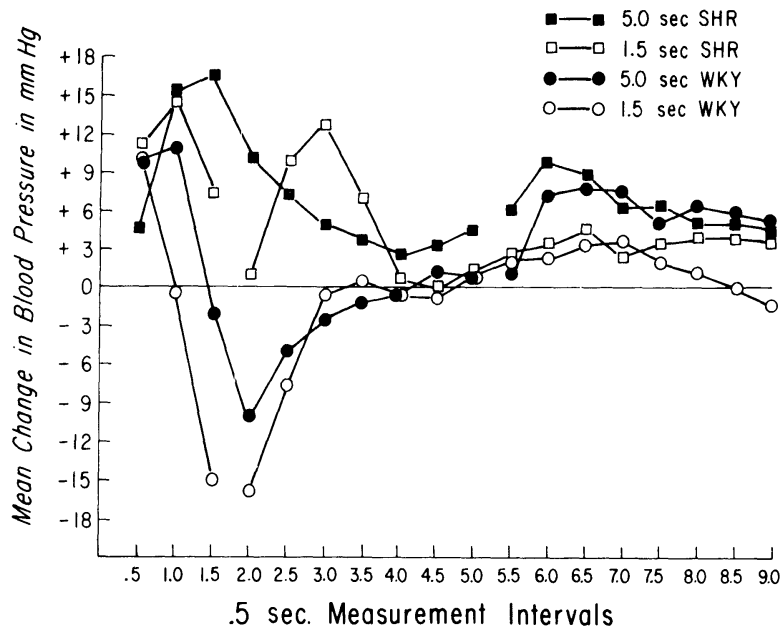

Figure 1. Mean change in blood pressure of each group in .5-sec intervals during and following shock averaged over the 12 shock trials.

of the 1.5-sec group. For the WKY groups, the depressor change reached a maximum immediately after shock termination in the 1.5 -sec group and in the middle of shock in the 5 -sec group. The magnitude of the depressor reaction of the 1.5-sec group was larger than that of the 5.0-sec group. Following shock, the 5.0-sec WKY group showed a pressor change comparable to that occurring in the 5.0 -sec SHR group.

A three-way (strain $\times$ shock duration $\times$ trial blocks) analysis of variance, using average BP scores calculated over the measurement intervals during each shock (intervals .5 through 1.5 for the 1.5 -sec groups and .5 through 5.0 for the 5 -sec groups) as the raw data, provided a significant strain effect $[F(1,27)=13.83, p<.001]$ and a significant duration $x$ trials interaction $[F(3,81)=5.34, p<.01]$. The interaction was produced by the fact that the magnitude differences between the short- and long-shock groups within each strain were larger on initial trials than on later trials. Separate three-way (strain $\times$ trial blocks $\times$ measurement intervals for each shock duration) analyses, using each point during shock as the raw data, established that the forms or topographies of the responses of the two strains were significantly different from each other during the $1.5-\mathrm{sec}$ shock $[\mathrm{F}(2,28)=4.53, \mathrm{p}<.05]$ and during the 5 -sec shock $[F(9,117)=4.10, \mathrm{p}<.01]$. In the case of the 5 -sec comparison, the strain difference was more pronounced on the early trials than on later trials $[F(27$, $351)=1.51, p<.05]$. A four-way (strain $\times$ shock duration $\times$ trial blocks $\times$ measurement intervals) analysis on the eight measurement intervals immediately following shock in Figure 1 (i.e., Intervals 2.0 through 5.5 for the 1.5-sec groups and 5.5 through 9.0 for the 5 -sec groups) provided a significant strain effect $[F(1,27)=11.72]$ and a significant strain $\times$ 
duration interaction $[\mathrm{F}(1,27)=4.32, \mathrm{p}<.05]$. The interaction was due to the presence of a larger postshock difference between the two WKY groups than between the two SHR groups.

\section{Heart Rate}

The HR responses of the four groups averaged over all 12 shock trials are depicted in Figure 2. The responses of both WKY groups and of the 1.5-sec SHR group were similar and consisted of cardioaccelerations during and following shock. In contrast, the 5-sec SHR group showed a brief period of HR deceleration to shock onset followed by a relatively low level of $H R$ acceleration. The deviant response pattern of the 5-sec SHR group led to a significant strain $\times$ duration interaction $[F(1,27)=5.89$, $\mathrm{p}<.05]$ for mean HR among all groups during shock and to a significant strain $\times$ measurement intervals interaction between the 5-sec SHR and WKY groups. No other factors involving strain or duration were significant.

\section{Blood Pressure and Heart Rate Correlations}

Pearson product-moment correlation tests were used to compare baseline BP and HR and the BP and $H R$ responses during each measurement interval averaged over the 12 shock trials. The outcomes of the tests for each group are displayed in Table 1. Only the 5.0-sec WKY group displayed a significant correlation between baseline BP and HR. With the exception of the 1.5-sec SHR group, none of the groups showed significant correlations during the early shock periods when major changes in BP were occurring. A cluster of correlations was found for three of the groups during Intervals 2.5 through 4.0. For the 1.5-sec SHR and WKY groups, BP was gen-

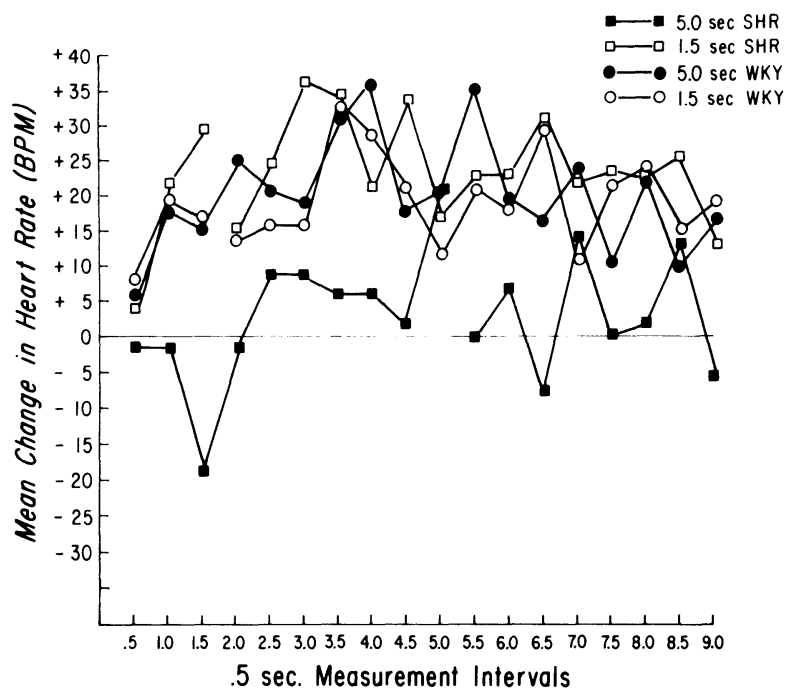

Figure 2. Mean change in heart rate of each group in .5-sec intervals during and following shock averaged over the 12 shock trials.
Table 1

Correlation Tests Between Blood Pressure and Heart Rate in .5-Sec Intervals Averaged Over the 12 Shock Trials

\begin{tabular}{lcccc}
\hline & \multicolumn{4}{c}{ Group } \\
\cline { 2 - 5 } Interval & 1.5 & 5.0 & 1.5 & 5.0 \\
\hline Baseline & .34 & .40 & .52 & $.85^{*}$ \\
.5 & $-.71^{*}$ & .09 & .51 & .12 \\
1.0 & -.58 & -.26 & .37 & -.35 \\
1.5 & .60 & -.11 & -.13 & -.16 \\
2.0 & .23 & -.40 & -.61 & -.38 \\
2.5 & $.71^{*}$ & $-.79^{*}$ & .20 & .64 \\
3.0 & $.78^{*}$ & .36 & $.82^{*}$ & -.22 \\
3.5 & $.82^{*}$ & $-.78^{*}$ & $.72^{*}$ & .45 \\
4.0 & .27 & $-.76^{*}$ & -.42 & .43 \\
4.5 & .18 & -.43 & $.81^{*}$ & .33 \\
5.0 & .33 & -.41 & .36 & .17 \\
5.5 & .34 & -.60 & -.22 & .22 \\
6.0 & .57 & .08 & .65 & .12 \\
6.5 & .36 & -.66 & .35 & -.15 \\
7.0 & .55 & .02 & .59 & $.91^{*}$ \\
7.5 & .70 & -.40 & .31 & -.23 \\
8.0 & .55 & .11 & .58 & -.26 \\
8.5 & $1.00^{*}$ & .73 & .30 & -.13 \\
9.0 & .40 & -.31 & .65 & .24 \\
\hline
\end{tabular}

*Significant at $p<.05$ level.

erally increasing during this time, with BP magnitude being positively related to HR acceleration. For the 5 -sec SHR group, the correlations were associated with decreasing BP and increased $H R$ in an inverse relationship (i.e., large $B P$ scores occurring with small HR scores).

\section{Baselines}

Mean baseline BP of the combined SHR groups was significantly higher than that of the combined WKY groups $(160$ vs. $141 \mathrm{~mm} / \mathrm{Hg})[\mathrm{F}(1,27)=18.82$, $\mathrm{p}<.01$ ]. Within the SHR strain, the 5-sec group had higher baseline BP than did the 1.5-sec group (169 vs. $152 \mathrm{~mm} / \mathrm{Hg}$ ), which led to a reliable strain $\times$ duration interaction $[F(1,27)=4.36, p<.05]$. For HR, the baseline of the combined WKY groups was significantly higher than that of the combined SHR groups $(458$ vs. $422 \mathrm{bpm})[\mathrm{F}(1,27)=5.49, \mathrm{p}<.05]$.

\section{DISCUSSION}

In keeping with findings of an earlier study (Hatton et al., 1981), the BP responses of the WKY groups to shock had both pressor and depressor components, while those of the SHR groups were mainly pressor changes. The current experiment demonstrated that the depressor activity was initiated in the WKY strain during shock, following a brief pressor reaction to shock onset. In the 5-sec WKY group, the depressor change was reversed in the middle of shock, with BP then returning toward baseline during the last half of the shock interval. Although both SHR groups displayed BP decreases subsequent to shockonset pressor responses, the decreases were more 
gradual than those of the WKY groups, and SHR BPs remained above baseline.

The fact that BP first increased and then decreased in all groups regardless of shock duration suggests that the decreases were mainly secondary reflex adjustments initiated by the pressor reactions to shock onset and that, once generated, were relatively independent of the stimulation provided by further shock. While it is true that the BP decreases were attenuated slightly by the continued presence of shock in both 5 -sec groups, especially on early trials, the response topographies of the short- and long-shock groups within each strain were highly similar to each other. In the case of the WKY strain, the similarity was particularly striking with respect to the return of BP toward baseline after the depressor reaction. Both WKY groups showed the same recovery pattern, even though for the 5-sec group shock was still being applied. For the SHR strain, both groups showed shockoffset pressor changes that were only slightly smaller following 5 -sec of shock than following $1.5 \mathrm{sec}$ of shock.

Baroreceptor modulations of sympathetic tone provide one reflex mechanism by which the observed BP oscillations to shock may have been controlled. The initial pressor responses of both strains would be expected to trigger increased baroreceptor activity, which in turn would lead to a reduction in sympathetic tone, causing BP to fall. The fact that the fall in pressure was more precipitous in the WKYs than in the SHRs could be accounted for on the basis of results reported by Judy, Watanabe, Henry, Besch, Murphy, and Hockel (1976). They found that baroreceptor activation produced less inhibition of sympathetic nerve activity in the SHR strain than in normotensive controls. The authors proposed that SHRs may have a central component of sympathetic activity that is not inhibited by baroreceptor stimulation. A similar possibility raised by Hallback and Folkow (1974) that might also apply in the current experiment is that baroreceptor reflexes may be more susceptible to central inhibitory influences in SHRs than in normotensive rats during stressful stimulation.

One problem with the notion of major baroreceptor involvement in the BP perturbations that occurred subsequent to shock onset is that only the 5sec SHR group displayed the decelerative HR changes that would be expected on the basis of increased baroreceptor activity. The other groups showed HR accelerations, and the levels of HR did not appear to vary systematically with changes in BP. Most of the correlations between HR and BP that were found occurred 2 to $4 \mathrm{sec}$ after shock onset, which was generally subsequent to the pressor decreases. Moreover, except for the 5-sec SHR group, the correlations reflected positive relationships between BP and HR rather than the inverse relationships that are typical of baroreceptor activity, suggesting that differences in general reactivity were being sampled by the correlations.

Evidence has been offered by Hatton et al. (1981) and Yamaguchi \& Kopin (1980) that SHR rats in the labile phase of hypertension may have impaired $\beta_{2}$-adrenoceptor-produced vasodilation. However, failure of the BP decreases of the SHR groups in the current experiment to match those of the WKY groups cannot be explained on that basis, since the latencies of the beginning of the BP decreases were too short (Coleman, 1980) to be mediated by circulating catecholamine stimulation of $\beta_{2}$ activity. It can also be noted in this regard that the depressor activity cannot be attributed to active vasodilation due to sympathetically mediated cholinergic peripheral vasodilation, since this mechanism is apparently absent in rats (Bolme, Novotny, Uvnas, \& Wright, 1970).

Except for the higher baseline HR of the WKYs over the SHRs, a strain difference that seems to vary unpredictably among studies (Kvetnansky, McCarty, Thoa, Lake, \& Kopin, 1969; McCarty, Chiueh, \& Kopin, 1978), the present findings are consistent with a growing number of reports of high sympathetic tone in SHR rats (Hallback et al., 1977; Iriuchijima, 1973; Judy et al., 1976; McCarty, Kvetnansky, Lake, Thoa, \& Kopin, 1978). Here, however, the elevated tone was indexed by less sympathetic withdrawal relative to that of the WKYs following a brief period of increased sympathetic activity associated with the shock-onset pressor reactions. The magnitudes of the onset reactions themselves were comparable in the two strains.

Although the exact mechanisms underlying the observed strain differences in sympathetic restraint cannot be specified, it seems likely that central cardiovascular pathways were involved in the occurrence of the biphasic BP changes shown by both strains. For example, Scherrer (1962) found that a transient increase in sympathetic activity caused by hypothalamic stimulation could result in postexcitatory inhibition of sympathetic outflow. These changes in sympathetic outflow were associated with a biphasic BP response not unlike that observed in the present study. Scherrer's results suggested that the depressor component was relatively independent of feedback from the vasculature but could be augmented by baroreceptor activity.

\section{REFERENCES}

Bolme, P., Novotny, J., Uvnas, B., \& Wright, P. G. Species distribution of sympathetic cholinergic vasodilator nerves in skeletal muscle. Acta Physiologica Scandinavica, 1970, 78, 60-64.

Coleman, T. G. Arterial baroflex control of heart rate in the conscious rat. American Journal of Physiology, 1980, 238, H515-H520. 
Hallback, M., \& Folkow, B. Cardiovascular respunses to acute mental "stress" in spontaneously hypertensive rats. Acta Physiologica Scandinavica, 1974, 90, 684-698.

Hallback, M., \& Weiss, L. Mechanisms of spontaneous hypertension in rats. Medical Clinics of North America, 1977, 61, 593-609.

Hatton, D. C., Buchholz, R. A., \& Fitzgerald, R. D. Autonomic control of heart rate and blood pressure in spontaneously hypertensive rats during aversive classical conditioning. Journal of Comparative and Physiological Psychology, 1981, 95, 978-990.

IrIUChiJima, J. Sympathetic discharge rate in spontaneously hypertensive rats. Japanese Heart Journal, 1973, 14, 350-356.

Judy, W. V., Watanabe, A. M., Henry, D. P., Besch, H. R., MurPhy, W. R., \& Hockel, G. M. Sympathetic nerve activity: Role in regulation of blood pressure in the spontaneously hypertensive rats. Circulation Research, 1976, 38(6, Suppl. 2), 11211129.

Kvetnansky, R., McCarty, R., Thoa, T. B., Lake, C. R., \& KopIN, I. J. Sympatho-adrenal responses of spontaneously hypertensive rats to immobilization stress. American Journal of Physiology, 1969, 5, H457-462.

McCarty, R., Chiveh, C. C., \& Kopin, I. J. Spontaneously hypertensive rats: Adrenergic hyperresponsivity to anticipation of electric shock. Behavioral Biology, 1978, 23, 180-188.

McCarty, R., Kvetnansky, R., Lake, C. R., Thoa, N., \& KopIN, I. Sympatho-adrenal activity of SHR and WKY rats during recovery from forced immobilization. Physiology \& Behavior, 1978, 21, 951-955.

ScherRer, H. Effect of basal blood pressure levels on blood pressure responses to central nervous stimulation. Annals of the New York Academy of Sciences, 1962, 98, 1302-1315.

YAmAGUChI, I., \& KoPIN, I. J. Blood pressure, plasma catecholamines and sympathetic outflow in pitched SHR and WKY rats. American Journal of Physiology, 1980, 238, H365-H372.

(Manuscript received June 12, 1981; accepted for publication September 14, 1981.) 